Potravinarstvo Slovak Journal of Food Sciences vol. 13, 2019, no. 1, p. 65-75

https://doi.org/10.5219/1020

Received: 18 January 2019. Accepted: 28 January 2019. Available online: 28 February 2019 at www.potravinarstvo.com

\title{
BIOINFORMATICS ANALYSIS OF AFLATOXINS PRODUCED BY ASPREGILLUS SP. IN BASIC CONSUMER GRAIN (CORN AND RICE) IN SAUDI ARABIA
}

\author{
Latifa Al Husnan, Muneera Al Kahtani, Randa Mohamed Farag
}

\begin{abstract}
The food contaminants by aflatoxins are inevitable even when all precautions and good agricultural practices are applied. Samples of white rice and corn (yellow, red) grains were collected from different local markets and houses. Three Aspergillus flavus strain isolated were identified using molecular characterization of $A F L R(a f l R)$ toxin gene. DNA genome of the three A. flavus isolates (namely A. flavus_ YC; A. flavus_ RC; A. flavus_ Rice) which corresponds to isolates from, yellow corn, red corn and white rice respectively were used as a template for PCR to amplify Aspergillus flavus AFLR (aflR) toxin gene. Partially sequenced was amplified using a specific primer set to confirm its identity, phylogenetic relationships between the three isolates as well as determination of the corresponding antigenic determinants. The epitope prediction analysis demonstrated that there were $1,2,3$ and 4 epitopes whose score were equal 1 in A.flavus_ YC; A. flavus_ RC; A. flavus _ Rice, respectively. Interestingly, there were great dissimilarity in the epitope sequences among the three isolates except in RLQEGGDDAAGIPA, SPPPPVETQGLGGD, RPSESLPSARSEQG and PAHNTYSTPHAHTQ were found to be similar between all isolates. This work articulates that the molecular identification and characterization of three A. flavus using Aspergillus flavus AFLR (aflR) toxin gene and the unique antigenic determinants that could be used for design of a broadspectrum antibody for rapid detection of $A$. flavus in foods and support quality system of food safety.
\end{abstract}

Keywords: PCR; sequences; phylogenetic tree; protein toxic gene; antigenic determinants

\section{INTRODUCTION}

Fungi caused major crops diseases during harvest and storage under higher temperature and humidity conditions (Bhat et al., 2010; Pasquali et al., 2016). While more than 25 different fungi species known to invade stored grains and legumes (Duan et al., 2007) some species such as Aspergillus, Fusarium, Penicillium are responsible for most spoilage and germ damage during storage (Boutigny et al., 2012; Aamot et al., 2015; Kachapulula et al., 2017). Crop transfers through international trade have made aflatoxins contaminated food a worldwide problem (Passone et al., 2010). Mycotoxins are secondary metabolites produced by fungi, which cause health hazards to animals and human beings; the majority of mycotoxins of greatest concern to human and animal health are produced by the genera Aspergillus, Penicillium, and Fusarium, the so-called field fungi that frequently infect various food commodities (Reddy et al., 2010). Toxicity of a mycotoxin will be manifested by its effect on the human and animal health and productivity of crops (Abdel-Wahhab et al., 2006). The main routes of mycotoxins exposure are ingestion, inhalation or through skin contact. The toxicity of a mycotoxin is determined by metabolism involving the, transformation, administration, distribution, absorption, excretion and molecular interactions of the toxin and its metabolites. Nowadays the main mycotoxins of interest are aflatoxins (AFs), ochratoxins, (Frisvad et al., 2019), trichothecenes, zearalenone, fumonisins, ergot alkaloids and deoxynivalenol (Reddy et al., 2010; Cendoya et al., 2014; Covarelli et al., 2015; Singh and Cotty, 2019). Mycotoxin producing fungi which are associated with groundnuts, peanuts, cereals such as maize, rice, sorghum, wheat, barley and oats and spices such as black pepper, ginger, nutmeg, chilly, etc. are considered to be of greater significance for all over world (Kumar et al., 2008; Brožková et al., 2015). Several studies have revealed mycotoxin contamination in rice worldwide: for example, aflatoxins in the United Arab Emirates (Osman et al., 1999) fumonisins in Iran, Argentina (Alizadeh et al., 2012; Cendoya et al., 2014) OTA in Morocco (Juan et al., 2008), ZEA in Nigeria (Makun et al., 2007), DON in Italy (Lorè et al., 2011) nivalenol in Korea (Lee et al., 2011) and citrinin in Egypt (Abd Allah and Ezzat, 2005). As most of the corn and rice is grown during the wet season; it is 
susceptible to mycotoxin contamination. Rice is shown to be a good substrate for toxigenic fungi like A. flavus, A. ochraceus, Penicillium citrinum, and $F$. proliferatum (Aamot et al., 2015; Arino et al., 2007; Sánchez-Hervás et al., 2008). Humidity, temperature, storage conditions, and transportation period are the factors that influence mycotoxin production in rice (Ariño et al., 2007). Regarding legumes in Saudi Arabia, very little information exists with respect to its natural contamination with toxigenic fungi and mycotoxins. Aflatoxins were detected in some Aspergillus isolates while fumonisin was detected in some Fusarium isolates (Ibrahim et al., 1998; AbdelFatah et al., 2017). Among food contaminants, mycotoxins may cause substantial economic loss due to lower availability of commodities with acceptable levels of mycotoxins present and possibly greater cost of mycotoxinsafe and acceptable foods (Mwanza et al., 2013; Samina, 2015). Mycotoxins continue to pose various health risks to consumers depending on specific mycotoxin consumed and level of exposure, and health status of individuals in the population (Voss et al., 2014; Pasquali et al., 2016). Many human diseases, especially carcinogenic, teratogenic, hepatic, and gastrointestinal ones, have been found linked with the ingestion of mycotoxin-contaminated products (Fung and Clark, 2004; Shephard, 2008; Samina, 2015). Outbreaks of mycotoxicoses in humans and animals, caused by ingestion of products containing mycotoxins, (Peraica and Rašić, 2012). Risk assessments relating to food safety are frequently hampered by the lack of quantitative data (Schmidt-Heydt et al., 2007). The sequencing fungal genomes and the studies of the molecular basis of fungal pathogenicity provide the study of risk factors associated with continuous exposer of mycotoxins (Bilodeau, 2011; Taha et al., 2012). This paper was shown concentrated on aflatoxins produced by Aspregillus sp. and possibility for used the similarity between amino acid toxin gene of Aspregillus isolates strain for produced antibodies. Where the database of Aspregillus genomes provides a comprehensive resource of genomics data information's an important plant and human pathogenic fungal genus Aspregillus. It's given useful for discovery of genes encoding industrial enzymes, and antibiotics which may control in aflatoxin food contaminations (Spröte et al., 2009; Taha et al., 2012). AFs are a group of polyketidederived furanocoumarins, with at least 16 structurally related toxins that have been characterized. These toxins are produced by a number of different Aspergillus species are primarily produced by Aspergillus flavus and Aspergillus parasiticus (Geiser et al., 2007; Ito et al., 2001). There are four major AFs (AFB1, AFB2, AFG1, AFG2) all of which occur naturally (Abbas et al., 2010). AFB1 is the most commonly occurring of the mold producing compounds (Abdulkadar et al., 2004). AFB1 has been included in category $1 \mathrm{~A}$ of active carcinogenic compound (Abou-zeid et al., 1997; Abdel-Wahhab et al., 2006). Factors influencing the presence of mycotoxins in foods or feeds include environmental conditions related to storage that can be controlled (Park et al., 2005). So, in this study, we have hypothesized that mycotoxins effects in human populations year to year because other factors as the fungal strain specificity, strain variation, and instability of toxigenic properties are more difficult to control (El-Manzalawy et al., 2008a; El-Manzalawy et al., 2008b). However, by the articulate the molecular identification and characterization of mycotoxins, can be control in mycotoxins effects. In this study our aim advocate to using molecular detection and bioinformatics characterization for study the properties of protein toxin gene of aflatoxins in main consumer grains as rice and corn. Many human diseases occurring in Japan and other Asian countries were attributed to mycotoxins after consumption of mold-damaged rice (Taligoola et al., 2011). Unfortunately, enactment of stringent rules for mycotoxin control in food is not always the best solution (Samina, 2015). The impact of mycotoxin standards is more drastic for the population of developing countries (Pasquali et al., 2016; Yassin et al., 2010).

\section{Scientific hypothesis}

Therefore, the aim of this study was to determine the Aspergillus species by the molecular identification of toxigenic mycotoxin profiles of those species that are naturally occurring in contaminating corn and rice seeds (as the main crops imported in Saudi Arabia) and protein structural analysis depicted from the gene(s) responsible for toxin biosynthesis. Which can be used as screening test for cost-effective control of mycotoxin and their products. We have hypothesized that by study the molecular characterizations and bioinformatics properties of Aflatoxins could in future be able to produce vaccine for species of Aspergillus genera which have higher prevalence rate in development countries (Bhatnagar et al. 2003; Pasquali et al. 2016).

\section{MATERIAL AND METHODOLOGY}

\section{Grains samples and isolation of mycotoxigenic} Aspergillus species

One hundred fifty grains corn (yellow and red grains) and rice were collected from different area of Saudi Arabia (Riyadh, Hail, Qasim, Asir,Tabuk, Jizan, Jouf, Jeddah and Dammam), where collected from storage markets and houses. The collected grains were randomly and its weight between $0.5-1 \mathrm{~kg}$ of each grain in cleans and dries packaging. Agar plate and blotter tests were used to isolate Aspergillus sp. as described by Neergaard (1977). Grains were divided into two groups, the first group was disinfected with sodium hypochlorite $1 \%$ for 2 min and the second group was non-disinfected. All grains were washed several times by sterilized water, and then dried between sterilized filter papers. The half of each group was plated on potato dextrose agar (PDA) (Sigma-Aldrich, USA). All dishes were incubated for $5-7$ days at $25{ }^{\circ} \mathrm{C}$. All isolation process under sterilized conditions to prevent any contamination of grain.

\section{Purification and identification of Aspergillus species}

Aspregillus species were initially identified to species based on the morphological characteristics (Leslie, Summerell and Bullock, 2006) of the macroconidia, microconidia and general mycelium presentation from a single spore isolate grown for 7 - 10 days on SNA with an Olympus BH-2 (Olympus America, New York) light microscope. Potato Dextrose Agar (PDA) was used to identify colony pigment characteristics of aerial mycelium on the agar (Leslie, Summerell and Bullock, 2006). 
Carnation Leaf Agar (CLA) (bio-WORLD, USA) was used to identify macroconidia, chlamydospores and the presentation of aerial mycelium single colony was transferred and purified by hypha tip technique onto PDA medium in the presence of streptomycin $\left(50 \mathrm{mg} . \mathrm{ml}^{-1}\right)$. The developing fungi were prepared for molecular identification using primers specific for the Aspergillus flavus AFLR (aflR) toxin gene. All conditions of isolation and purification of mycotoxins were performed under sterilization for prevent any external agent of seeds pollutions.

\section{Molecular identification of Aspergillus flavus AFLR (aflR) toxin gene \\ Isolation of DNA genome}

The mycelium mass of Aspergillus species isolates grown on PDA broth medium was harvested by centrifugation at $6000 \mathrm{rpm}$ for $10 \mathrm{~min}$. The pellets were washed twice by PBS buffer and stored at $200{ }^{\circ} \mathrm{C}$. Total DNA of the three isolates was isolated using lysozyme - dodecyl sulfate lysis method as described by Leach et. al. (1990).

\section{Amplification and purification of Aspergillus flavus AFLR (aflR) gene}

Specific PCR reactions were conducted to assess the presence of AFLR (aflR) gene. The primers used as described by Cary et al. (2000) were: Omtl-F (aflR) (5'-GCCTTGCAAACACACTTTCA-3'); Omtl-R 5'-AGTTGTTGAACGCCCCAGT3') and optimal annealing $\left(\mathrm{Tm}=55^{\circ} \mathrm{C}\right)$. The PCR amplification conditions included initial denaturation at $94{ }^{\circ} \mathrm{C}$ for 5 min then 35 cycles at $94{ }^{\circ} \mathrm{C}$ for $30 \mathrm{~s}, 55{ }^{\circ} \mathrm{C}$ for $60 \mathrm{~s}$ followed by extension step at $72{ }^{\circ} \mathrm{C}$ for $90 \mathrm{~s}$. and a final extension at $72{ }^{\circ} \mathrm{C}$ for $7 \mathrm{~min}$. The amplification reaction was performed by thermal cycler (Applied Biosystems, USA). Purification of PCR product was detected by electrophoresis (PNU, Faculty of science, Research center) using agarose $1.5 \%$ in 1x TAE buffer and staining with ethidium bromide (Qiagen, Berlin, Germany) (Sambrook et al., 1989). The resultant fragment of Aspergillus flavus AFLR (aflR) toxin gene was excised from the gel and purified using a QIA quick gel extraction kit (Qiagen, Berlin, Germany).

\section{DNA sequencing}

The purified PCR products were prepared for Sanger sequencing technology using DNA sequencer technique (Sigma, central lab, PNU, KSA). DNA sequences of Aspergillus flavus isolates were aligned using Bio Edit software version 7 (www. Mbio-NCUs. Edu/bio. Edit) and were compared of the often accessions of Aspergillys sp. available in the NCBI data base using BIASI- algorithm to identify closely related sequences (http/WWW.NCbI.Nih.Gov). Dendrograms were constructed by using unwerighted pair Group method with Arithmetic (UPGMA) on Gen bank.

\section{Epitope prediction and antigenicity}

The primary amino acids sequence of the Aspergillus flavus AFLR (aflR) toxin gene protein was evaluated from the corresponding nucleotide sequence using MEGA 6.0 software. The linear B-cell epitopes in the primary amino acid sequence of the coat protein was performed using
BCPREDS server with default parameters (http://ailab.cs.iastate.edu/bcpreds/) which implements a support vector machine (SVM) and the subsequence kernel method (El-Manzalawy et al., 2008a). Flexible length linear B-cell epitopes were predicted using FBCP red (El-Manzalawy et al., 2008b) method with a specificity cut-off; $75 \%$. The antigenicity of each amino acid residue in the primary protein sequence was determined using a semi-empirical method (Kolaskar and Tongaonkar, 1990) which makes use of physicochemical properties of each amino acid and their frequencies of occurrence in experimentally known segmental epitopes.

\section{RESULTS AND DISCUSSION}

Three Aspergillus isolates from tested grains by PDA method was purified by single spore and hypha tip on PDA slant medium. The Aspergillus isolates were selected for molecular identification using Aspergillus flavus AFLR (aflR) toxin gene sequencing. Three Aspergillus isolates represented grains from yellow corn, red corn and white rice and designated as A. flavus _ YC; A. flavus _ RC; A. flavus _ Rice respectively.

\section{Molecular characters of toxin gene}

Total DNA was extracted from A. flavus _ YC; A. flavus _ RC; A. flavus _ Rice isolates infected grains. Aspergillus flavus AFLR (aflR) toxin gene was amplified from isolated DNA of mycelium using PCR reaction mixture and specific primer sets. PCR amplicons were allowed for sequencing reaction through cycle sequencing method. The DNA amplicons returned as electropherogram files. Electropherogram showed distinct peaks for each base cell as well as high $Q$ values for each cell. Sequences obtained for each primer for each isolate had sufficient overlap between them and used to form one continuous sequence (Coting). The nucleotide partial sequence of Aspergillus flavus AFLR (aflR) toxin gene in the three isolates was compared with published isolates on Gen Bank. The sequence homology revealed that the gene of interest Aspergillus flavus AFLR (aflR) toxin gene and the test fungal isolates was Aspergillus flavus isolates. A multiple sequence alignment was constructed using ClustalW software (GNU Lesser GPL) between the three studied isolates. The alignment showed many conserved regions in all sequences as well as distinguished the heterogeneity positions among the aligned sequences (Figure 1a, 1b, 1c). Phylogenetic analysis was performed by construction of phylogenetic tree using a neighbor joining method to unravel the relationships among all Aspergillus flavus isolates (Figure 2). The phylogenetic tree resulted in two clades in which A. flavus _ Rice (white rice isolate) and A. flavus _ RC (red corn isolate) were in the same cluster whilst A. flavus _ YC (yellow corn isolate) was separate in a different cluster (Figure 2). Thus, the molecular identification based on sequence homology of the Aspergillus flavus AFLR (aflR) toxin gene confirmed the identity and phylogeny of the studied three Aspergillus flavus isolates.

The epitope prediction analysis demonstrated that there were 1, 2, 3 and 4 epitopes whose score were equal 1 in $A$. flavus _ YC (yellow corn), B: A. flavus _ RC (red corn) and C: A. flavus _ Rice (white rice). Also, there were great 
variations in the epitope sequences among the three isolates except in RLQEGGDDAAGIPA, SPPPPVETQGLGGD, RPSESLPSARSEQG and PAHNTYSTPHAHTQ were found to be common between all isolates. These residues with high frequencies of occurrences in antigenic determinants were highlighted (yellow) in the antigenicity profile (Figure 3). Figure (3) also show the variability in the positions and types of amino acid residues with high antigenic frequency.

Mycotoxin detection is a major problem in developing countries where contaminated food commodities may readily reach food stores and homes (Bilodeau, 2011; Boutigny et al., 2012; Taha et al., 2017). The risk of contamination by mycotoxins is an important food safety concern for grains and other field crops (Abdulkadar et. al., 2004; Mwanza et al., 2013). Humans are exposed to mycotoxins throughout their life time due to consumption of fungus-contaminated food products and many human diseases, especially carcinogenic, teratogenic, hepatic, and gastrointestinal ones, have been found linked with the ingestion of mycotoxin-contaminated products (Fung and Clark, 2004; Shephard, 2008; Mwanza et al., 2013). Sufficient quantities of mycotoxins in food and feedstuff can adversely affect human and animal health (Qiu and Shi, 2014). Environmental factors and host species have a strong impact on the occurrence of a specific chemotype and the incidence of Aspergillus species (Bhatnagar et al., 2003). The distribution of Aspergillus species in maize is influenced by optimal climatic conditions, pathogenicity and competition between other fungi (Bhatnagar et al., 2006). The type of environmental factor identified in the incidence of Aspergillus species as demonstrated in recent EU maize surveys (Creepy, 2002). In those studies, the prevalence of species varied year-to-year and was believed to be associated with the differences in climatic conditions between years (Caldas et al., 2002; Scauflaire et al., 2011). As was reported by Covarelli et al. (2015) the emergence of toxigenic fungi on small grains has a negative impact on the safety and quality of feed and food. in this study We isolate mycotoxins strains of Aspergillus sp from contaminated corn and rice grains by a genomic library through amplication mycotoxins structure genes ( $a f l R$ ) by polymerase chain reaction (PCR) and sequencing of amplicans this result was agree with (Chen et al., 2002). Data clearly reveal that the PCR technique is efficient in distinguishing mycotoxins (Bilodeau, 2011; de Souza et al., 2005; Taha et al., 2012; Allam et al., 2015) from commonly inhabiting stored grains. We isolate Aspergillus sp. from both zellow and red corn which used in food and feed in human and animal; also isolated from long and short rice (Niessen, 2007; Fox and Howlett, 2008). Aspergillus species being able to grow at moderate to high temperature (Ehrlich et al., 2003) and its responsible for spoilage of food commodities during transport and storage. In addition, reduction in nutritive value, insipidness and discoloration are other problems resulted from contamination of grains by Aspergillus (Dean et al., 2012). Rapid and accurate identification of Aspergillus and/or their metabolites are mandatory for the implementation of preventive measures in the whole food production system as was reported by Bok and Keller, (2004); Bhatnagar et al. (2003) and Dean et al. (2012). The molecular characterization of three Aspergillus sp. isolated from small grains (yellow corn, white rice and red corn) using the mycotoxins gene, aflatoxin AFLR (aflR) allowed for coupled identification and mycotoxins screening in the three Aspergillus isolates (Bhatnagar et al., 2006). Mycotoxins-producing fungi were isolated from sorghum grains from Saudia Arabia before (Mahmoud et al., 2013; Yassin et al., 2010). Following the molecular identification of Aspergillus sp. Bcell epitopes in the aflatoxin $A F L R$ (aflR) gene were predicted. The characterization of B-cell epitopes using computational tools is highly advantageous for the synthesis of specific antibodies for rapid detection of microbial pathogens in their environments. The epitopes prediction saves labor and time for validation experiments. The identification of epitopes plays a crucial role in the vaccine design, immunodiagnostic testing and antibody production (Sette and Fikes, 2003). In this study, BCPREDS server was used to predict epitopes found in the primary amino acids sequence of aflatoxin $A F L R$ ( $a f l R$ ) protein. BCPREDS proved high efficiency to predict linear B-cell epitopes in SARS-CoV S protein (El-Manzalawy et al., 2008a; ElManzalawy et al., 2008b). There was variability in the sequence and numbers of epitopes among the three toxin proteins analyzed. Here, a fixed length of epitopes (14 residues) was observed. The epitope prediction analysis demonstrated that there were 1, 2, 3 and 4 epitopes whose score were equal 1 in A. flavus _ YC; A. flavus _ RC; A. flavus _ Rice respectively. In this study, there were great dissimilarity in the epitope sequences among the three isolates. But we found that there some epitope sequences were common between all isolates in RLQEGGDDAAGIPA, SPPPPVETQGLGGD, RPSESLPSARSEQG and PAHNTYSTPHAHTQ. This result suggesting its exploitation for design of a specific antibody to be used for rapid detection of different Aspergillus species in small grains (Ehrlich et al., 2003; Degola et al., 2007). The highly frequent residues with high antigenicity profiles such as valine, leucine, isoleucine, aspartic acid, glutamine and glutamic acid are mostly hydrophobic (Duan et al., 2007). The occurrence of hydrophobic residues in epitopes is frequent and do have a hierarchy signature (Zhang, 2002; Aftabuddin and Kundu, 2007; Suga and Galel, 2007; Scauflaire et al., 2011). Epitope prediction has many implications in pathogen detection and differentiation applications. The consideration of occurrence of Aspergillus sp. on small grains is important in risk assessment of mycotoxins and setting up preventive measures proactively. The main problems concentrated in high risk outbreaks of aflatoxicosis have been reported in different countries all over the world; that because the widespread of aflatoxin contamination especially in developing countries (Pitt 2000a; Pitt 2000b; Kovacs, 2004). No doubt, that the safety control in food and feed processing should be by prevention of mycotoxin contamination in agriculture by used as simple and rapid screening tests for cost-effective control of food diseases by production of vaccines (Sette and Fikes, 2003; Probst et al., 2014; Mwanza et al., 2013; Allam et al., 2015). 

A. flavus_YC
A. flavus RC
A. flavus_Rice

A. flavus YC

A. flavus RC

A. flavus_Rice

A. flavus YC

A. flavus $R C$

A. flavus_Rice
A. flavus_YC
A. flavus RC
A. flavus_Rice

A. flavus_YC

A. flavus RC

A. flavus_Rice

A. flavus YC

A. flavus RC

A. flavus_Rice
A. flavus_YC
A. flavus $\mathrm{RC}$
A. flavus_Rice

A. flavus_YC

A. flavus RC

A. flavus_Rice

A. flavus YC

A. flavus_RC

A. flavus_Rice
A. flavus_YC
A. flavus RC
A. flavus_Rice

A. flavus_YC

A. flavus RC

A. flavus_Rice

670

680

690

700

710

720

GAGTCGGCCCCACTACCACCGTTTCAGGCGCGCTATTGCTGCTTTPCGCTAGCACTACAA GAGTCGGCCCCACTACCACCGTTTCAGGCGCGCTATTGCTGCTTTTCGCTAGCACTACAA GAGTCGGCCCCACTACCACCGTTTCAGGCGCGCTATTGCTGCTTTTCGCTAGCACTACAA

730

740

750

760

770

780

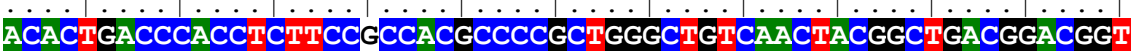
AСАСTGACCСАССТСTTCCCCCACGCCCGGCTGGGCTGTCAACTACGGCTGACGGACGGT ACACTGACCCACCTCTTCCCCCACGCCCCGCTGGGCTGTCAACTACGGCTGACGGACGGT

800

810

820

830

840

GAGGACA TTTCGTGCAACCTGATGACGACTGATATGGTCATCTCCGGGAACAÁGAGGGCT

GAGGACAGTTCGTGCAACCTGATGACGACTGATATGGTCATCTCGGGGAACAAGAGGGCT

GAGGACAGTTCGTACAACCTGATGACGACTGATATGGTCATCTCGGGGAACAAGAGGGCT

850

860

$870 \quad 880$

890

900

ACCGATGCGGTCCGGAAGATCCTCGGGTGTTCGTGCGCGCAGGATGGCTACTTGCTGAGC

ACCGATGCGGTCCGGAAGATCCTCGGGTGTTCGTGCGCGCAGGATGGCTACTTGCTGAGC

ACCGATGCGGTCCGGAAGATCCTCGGGTGTTCGTGCGCGCAGGATGGCTACTTGCTGAGC

910

920

930

940

950

960

ATGGTCGTCCTTATCGTTCTCAAGGTGCTGGCATGGTATGCTGCGGC̈ÁGCÁGCACCCÁG ATGGTCGTCCTTATCGTTCTCAAGGTGGTGGCATGGTATGCTGCGGCAGCAGGCACCCAG ATGGTCGTCCTTATCGTTCTCAAGGTGCTGGCATGGTATGCTGCGGCAGCAGGCACCCAG

980

990

1000

1010

1020

TGTACCTCAACGGCGGCGGGTGGAGAAACCAACAGTGCAGCTGTAGCAACÁTCCCGCC TGTACCTCAACGGCGGCGGGTGGAGAAACCAACAGTGGCAGCTGTAGCAACAGTCCCGCC TGTACCTCAACGGCGGCGÄTGGAGAAACCAACAGTGGCAGCTGTAGCAACAGTCCCGCC

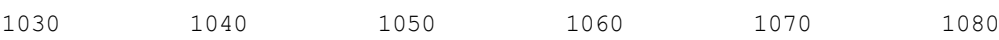

ACCGTGTCCA GTGGCTGTCTGACGGAÄAACCCETGCTGCACCTCCCTAGTATGATGGGC ACCGTGTCCAGTGGCTGTCTGACGGACGAGCGCGTGCTGCACCTCCCTAGTATGGTGGGC ACCGTGTCCAGTGGCTGTCTGACGGAAGAGCGCGTGCTGCACCTCCCTAGTATGGTGGGC
1090
1100
1110
1120
1130
1140

GAGGATTGTGTGGATGAGGAÄGACCÄGCGCGÄGGGCGGCACÁGTTGTTCTGAGTGAA GAGGATTGTGTGATGAGGAAGACCAGCCGCGAGTGGCGGCACAGCTTGTTCTGAGCGAI GAGGATTGTGTGGATGAGGAAGACCAGCCGCGAGTGGTGGCACAGCTTGTTCTGAGCGAA

1150

1160

1170

1180

1190

1200

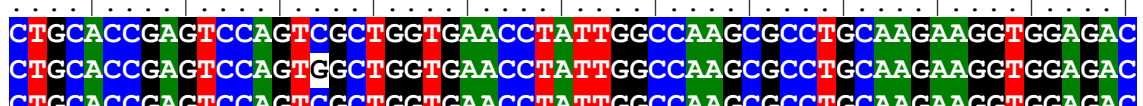

CTGCACCGAGTCCAGTCGCTGGTGAACCTATTGGCCAAGCGCCTGCAAGAAGGTGGAGAC

$\begin{array}{rrrrr}1210 & 1220 & 1230 & 1240 & 1250\end{array}$

GATGCAGCAGGGATACCGGCGCACCATCCAGCGTCCCGTTTCTCACTACTCGGGTTTAGT

GATGCAGCAGGGATACCGGCGCACCATCCAGCGTCCCCTTTCTCACTACTCGGGTTTAGT

GATGCAGCAGGGATACCGGCGCACCATCCAGCGTCCACTTTCTCACTACTCGGGTTTAGT

$\begin{array}{rrrrr}1270 & 1280 & 1290 & 1300 & 1310\end{array}$

GGCCTCGAAGCAAATCTCCGCCÄCCGTTTGCGCGCCGTGTCCTCCGACATTATTGATTAC

GGCCTCGAAGCAAATCTCCGCCAACGTTTGCGCGCCGTGTCTTCCGACATTATTGATTAC

GGCCTCGAAGCAAATCTCCGCCAACGTTTGCGCGCCGTGTCTTCCGACATTATTGATTAC

Figure 1b Multiple sequence alignment of the aflr gene partial sequence among three Aspergillus flavus isolated from yellow corn (YC), red corn ( $\mathrm{RC})$ and rice. 

A. flavus_YC
A. flavus $\mathrm{RC}$
A. flavus Rice

Figure 1c Multiple sequence alignment of the aflr gene partial sequence among three Aspergillus flavus isolated from yellow corn (YC), red corn ( $\mathrm{RC})$ and rice.

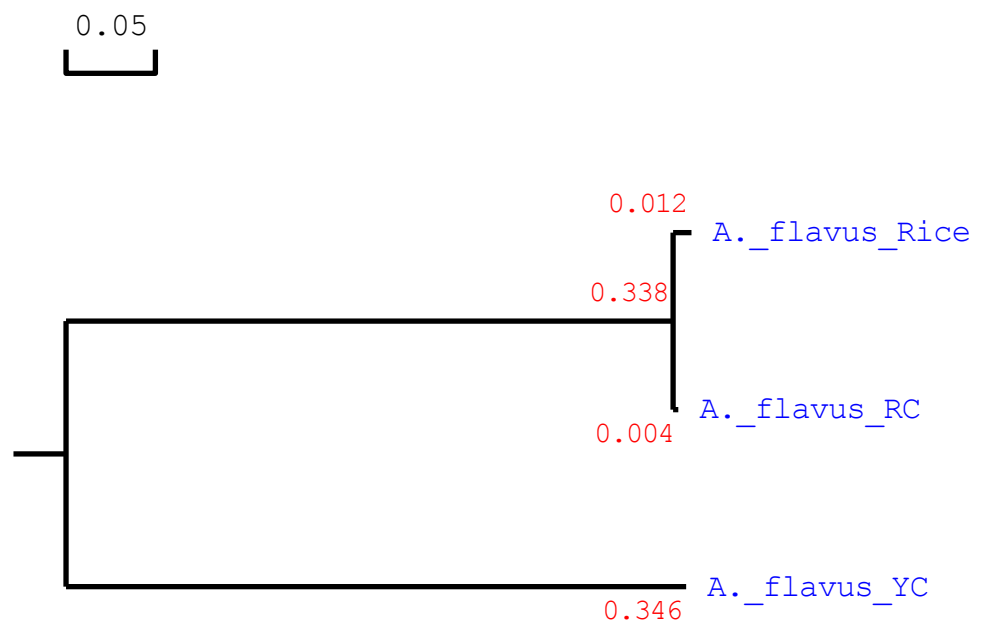

Figure 2 Phylogenetic tree using neighbor joining method among the three Aspergillus isolates.

Table 1 Flexible length predictions of epitopes in the amino acids sequence of $A F L R(a f l R)$ gene sequence protein of the three Aspergillus flavus isolates.

\begin{tabular}{|c|c|c|c|c|c|c|}
\hline No. & $\begin{array}{c}\begin{array}{c}\text { Epitope/ A. flavus _ YC } \\
\text { (yellow corn) }\end{array} \\
\end{array}$ & Score/ & $\begin{array}{c}\text { Epitope/ A. flavus _ RC } \\
\text { (red corn) }\end{array}$ & Score/ & $\begin{array}{c}\text { Epitope/ A. flavus } \\
\text { Rice (white rice) }\end{array}$ & Score/ \\
\hline 1 & RLQEGGDDAAGIPA & 1 & RLQEGGDDAAGIPA & 1 & RLQEGGDDAAGIPA & 1 \\
\hline 2 & SPPPPVETQGLGGD & 1 & SPPPPVETQGLGGD & 1 & SPPPPVETQGLGGD & 1 \\
\hline 3 & DHISPRASPGPIRS & 1 & DHISPRASPGPIRS & 1 & GETNSGSCSNSPAT & 1 \\
\hline 4 & PPHALPTPNGSSSV & 1 & PPHALPTPNGSSSV & 1 & RRASPGPIRSSQTR & 1 \\
\hline 5 & GETNSGSCSNSPAT & 1 & GETNSGSCSNSPAT & 1 & PHALPNRNGSSSVS & 1 \\
\hline 6 & IDPFFESAPLPPFQ & 0.997 & IDPFFESAPLPPFQ & 0.997 & IDPFLESAPLPPFQ & 0.996 \\
\hline 7 & MGRNPRAPSPLDST & 0.994 & MARNPRAPSPLDST & 0.995 & MGRNPRAPSPIDST & 0.992 \\
\hline 8 & RPSESLPSARSEQG & 0.99 & RPSESLPSARSEQG & 0.99 & RPSESLPSARSEQG & 0.99 \\
\hline 9 & PAHNTYSTPHAHTQ & 0.936 & PAHNTYSTPHAHTQ & 0.936 & PAHNTYSTPHAHTQ & 0.936 \\
\hline 10 & MEHGTHVDFLAEST & 0.847 & VRCTKEKPACARCI & 0.855 & VRCTKEKPACARCI & 0.855 \\
\hline 11 & SSGCLTEERVLHLP & 0.842 & TDGEDSSCNLMTTD & 0.79 & SSGCLTEERVLHLP & 0.842 \\
\hline 12 & TDGEDSSCNLMTTD & 0.79 & VVLIVLKVVAWYAA & 0.778 & THLFPHAPLGCQLR & 0.733 \\
\hline 13 & KVRCKEKPACARCI & 0.773 & VGEDCVDEEDQPRV & 0.713 & VGEDCVDEEDQPRV & 0.713 \\
\hline
\end{tabular}

\section{CONCLUSION}

A best strategy to control mycotoxins is only by prevention, because most mycotoxins are chemically stable, so they remain unaffected during storage and processing. For this reason, we believed that by using a highly sensitivity and selectivity methods as molecular identification and bioinformatics characterizations of protein toxic gene for mycotoxins gives chance for production of antibody against its toxicity. This paper was show that molecular identification in Aspergillus flavus in three isolates in small grains (rice and corn) with the epitope prediction analysis demonstrated that there were great differentiations in the epitope sequences among the three isolates except in four position (as described above) were found to be common between all isolates. This work articulates that the molecular identification and characterization of three A. flavus using Aspergillus flavus $A F L R$ (aflR) toxin gene and the unique antigenic determinants that could be used for design of a broadspectrum antibody for rapid detection of A. flavus in foods and feeds that conducive to control in aflatoxin levels and cover prevention of mycotoxins. 
A

\begin{tabular}{lcccccc}
1 & 11 & 21 & 31 & 41 & 51 & 60 \\
\hline & $\mid$ & $\mid$ & $\mid$ & $\mid$ & $\mid$ & $\mid$
\end{tabular}

MVDHISPRASPGPIRSSQTRRAGKLRDSCTSCASSKVRCKEKPACARCIERGLACQYMVS 60

. .EEEEEEEEEEEEEE . . . . . . . . . . . EEEEEEEEEEEEEE . . . . . . .

KRMGRNPRAPSPLDSTRRPSESLPSARSEQGLPAHNTYSTPHAHTQAHTHAHSHRQPHPQ 120

..EEEEEEEEEEEEEE.EEEEEEEEEEEEEE.EEEEEEEEEEEEEE ...........

SHPQSNQPPHALPTPNGSSSVSAIFSHQSPPPPVETQGLGGDLAGQEQSTLSSLTVDSEF 180

...... EEEEEEEEEEEEEE . . . . . EEEEEEEEEEEEEE . . . . . . . . . .

GGSLQSMEHGTHVDFLAESTGSLFDAFLEVGTPMIDPFFESAPLPPFQARYCCFSLALQT 240

......EEEEEEEEEEEEEE. . . . . . . . . EEEEEEEEEEEEEE . . . . . . .

LTHLFRHAPLGCQLRLTDGEDSSCNLMTTDMVISGNKRATDAVRKILGCSCAQDGYLLSM 300

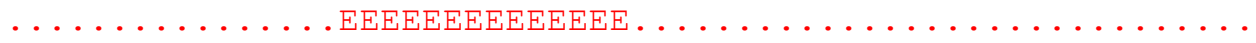

VVLIVLKVLAWYAAAAGTQCTSTAAGGETNSGSCSNSPATVSSGCLTEERVLHLPSMMGE 360

...................EEEEEEEEEEEEEE.EEEEEEEEEEEEEE....

DCVDEEDQPRVAAQLVLSELHRVQSLVNLLAKRLQEGGDDAAGIPAHHPASRFSLLGFSG 420

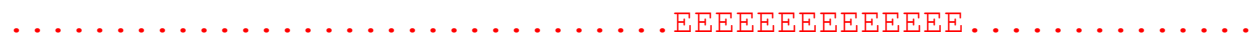

LEANLRHRLRAVSSDIIDYLHRE 443

..................

B

\begin{tabular}{lllllll}
1 & 11 & 21 & 31 & 41 & 51 & 60 \\
\hline & $\mid$ & $\mid$ & $\mid$ & $\mid$ & $\mid$ & $\mid$
\end{tabular}

MVDHISPRASPGPIRSSQTRRARKLRDSCTSCASSKVRCTKEKPACARCIERGFACQYMV 60

. .EEEEEEEEEEEEEE . . . . . . . . . . . . EEEEEEEEEEEEEE . . . . . .

SKRMARNPRAPSPLDSTRRPSESLPSARSEQGLPAHNTYSTPHAHTQAHTHAHSHPQPHP 120

...EEEEEEEEEEEEEE.EEEEEEEEEEEEEE.EEEEEEEEEEEEEE...........

QSHPQSNQPPHALPTPNGSSSVSAIFSHQSPPPPVETQGLGGDLAGQEQSTVSSLTVDSE 180

.......EEEEEEEEEEEEEE . . . . . EEEEEEEEEEEEEE . . . . . . . . . .

FGGSLQSMEHGNHVDFLAESTGSLFDAFLEVGTPMIDPFFESAPLPPFQARYCCFSLALQ 240

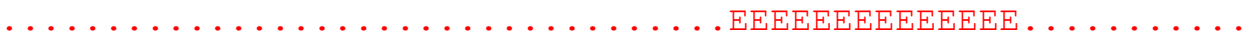

TLTHLFPHARLGCQLRLTDGEDSSCNLMTTDMVISGNKRATDAVRKILGCSCAQDGYLLS 300

.............EEEEEEEEEEEEEE . . . . . . . . . . . . .

MVVLIVLKVVAWYAAAAGTQCTSTAAGGETNSGSCSNSPATVSSGCLTDERVLHLPSMVG 360

. EEEEEEEEEEEEEE ..........EEEEEEEEEEEEEE . . . . . . ....EE

EDCVDEEDQPRVAAQLVLSELHRVQWLVNLLAKRLQEGGDDAAGI PAHHPAS PFSLLGFS 420

EEEEEEEEEEEE . . . . . . . . . . . EEEEEEEEEEEEEE . . . . . . . .

GLEANLRQRLRAVSSDIIDYLHRE 444

.................

C

$\begin{array}{ccccccc}1 & 11 & 21 & 31 & 41 & 51 & 60 \\ \mid & \mid & \mid & \mid & \mid & \mid & \mid\end{array}$

MVDHISRRASPGPIRSSQTRRARKLRDSCTSCASSKVRCTKEKPACARCIERGFACQYMV 60

......EEEEEEEEEEEEEE . . . . . . . . . EEEEEEEEEEEEEE. . . . . . .

SKRMGRNPRAPSPIDSTRRPSESLPSARSEQGLPAHNTYSTPHAHTQAHTHAHSHPQPHP 120

...EEEEEEEEEEEEEE.EEEEEEEEEEEEEE.EEEEEEEEEEEEEE ...........

QSHPQSNQPPHALPNRNGSSSVSAIFSHQSPPPPVETQGLGGDLAGQEQSTLSSLTVDSE 180

. . . . . . EEEEEEEEEEEEEE . . . . EEEEEEEEEEEEEE . . . . . . . . . . .

FGGSLQSMEHGNHVDF LAESTGS LFDAFMEVGTPMIDPFLESAPLPPFQARYCCFSLALQ 240

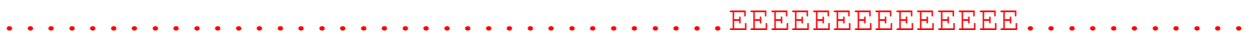

TLTHLFPHAPLGCQLRLTDGEDSSYNLMTTDMVISGNKRATDAVRKILGCSCAQDGYLLS 300

. EEEEEEEEEEEEEE . . . . . . . . . . . . . . . . . .

MVVLIVLKVLAWYAAAAGTQCTSTAASGETNSGSCSNSPATVSSGCLTEERVLHLPSMVG 360

...................EEEEEEEEEEEEEE.EEEEEEEEEEEEEE..EE

EDCVDEEDQPRVVAQLVLSELHRVQSLVNLLAKRLQEGGDDAAGIPAHHPASTFSLLGFS 420

EEEEEEEEEEEE . . . . . . . . . . EEEEEEEEEEEEEE . . . . . .

GLEANLRQRLRAVSSDIIDYLHREW 445

Figure 3 Show the amino acids sequence of $A F L R$ (aflR) gene sequence protein of the three Aspergillus flavus isolates. 


\section{REFERENCES}

Aamot, H. U., Ward, T. J., Brodal, G., Vrålstad, T., Larsen, G. B., Klemsdal, S. S., Elameen, A., Uhlig, S., Hofgaard, I. S. 2015. Genetic and phenotypic diversity within the Fusarium graminearum species complex in Norway. European Journal of Plant Pathology, vol. 142, no. 3, p. 501-519. https://doi.org/10.1007/s10658-015-0629-4

Abbas, H. K., Reddy, R. N., Salleh, B., Saad, B., Abel, C. A., Shier, W. T. 2010. An overview of mycotoxin contamination in foods and its implications for human health. Toxin Reviews, vol. 29, no. 1, p. 3-26. https://doi.org/10.3109/15569541003598553

Abd-Allah, E. F., Ezzat, S. M. 2005. Natural occurrence of citrinin in rice grains and its biocontrol by Trichoderma hamatum. Phytoparasitica; vol. 33, no. 1, p. 73-84. https://doi.org/10.1007/BF02980928

Abdel-Fatah, Sh. M., Noah Badr, A., Abu Sree, Y. H., Amar, H. A. 2017. Mycotoxigenic Fungi and Mycotoxins in Egyptian Barley under Climate Changes. Research J. Environm. Toxico. vol.11 no. 1, p. 1-10.

Abdel-Wahhab, M. A., Ahmed, H. H., Hagazi, M. M. 2006. Prevention of aflatoxin B1-initiated hepatotoxicity in rat by marine algae extracts. J. Appl. Toxicol., vol. 26, no. 3, p. 229238. https://doi.org/10.1002/jat.1127

Abdulkadar, A. H. W., Al-Ali, A. A., Al-Kildi, A. M., AlJedah, J. H. 2004. Mycotoxins in food products available in Qatar. Food Control, vol. 15, no. 7, p. 543-548. https://doi.org/10.1016/j.foodcont.2003.08.008

Abou-Zeid, A., Metwally, M., Farid, B. 1997. Physiological and hepatotoxic studies on fungal aflatoxin isolated from Egyptian cereals. Egyptian Journal of Microbiology, vol. 32, no. 1 , p. 83-98.

Aftabuddin, M., Kundu, S. 2007. Hydrophobic, hydrophilic, and charged amino acid networks within protein. Biophys. J., vol. 93, no. 1, p. 225-231. https://doi.org/10.1529/biophysj.106.098004

Alam, M., Chamhuri, S., Basri, T., Mazlin, M., Mohd, E. T. 2015. Climate change adaptation policy in Malaysia: Issues for agricultural sector. Braz. J. Microbiol., vol. 46 no. 2, p. 337346. https://doi.org/10.5897/AJARX11.030

Alizadeh, A. M., Rohandel, G., Roudbarmohammadi, S., Roudbary, M., Sohanaki, H., Ghiasian, S. A., Taherkhani, A., Semnani, S., Aghasi, M. 2012. Fumonisin B1 contamination of cereals and risk of esophageal cancer in a high-risk area in northeastern Iran. Asian Pac. J. Cancer Prev., vol. 13, no. 6, p. 2625-2628. https://doi.org/10.7314/APJCP.2012.13.6.2625

Ariño, A., Juan, T., Estopañan, G., González-Cabo, J. F. 2007. Natural occurrence of Fusarium species, fumonisin production by toxigenic strains, and concentrations of fumonisins B-1 and B-2 in conventional and organic maize grown in Spain. Journal of Food Protection vol. 2007, no. 70, p. 151-156. https://doi.org/10.4315/0362-028X-70.1.151

Ashiq, S. 2015. Natural occurrence of Mycotoxins in Food and Feed: Pakistan Perspective. Comprehensive Reviews in food science and food safety, vol. 14, no. 2, p. 159-175. https://doi.org/10.1111/1541-4337.12122

Bhat, R., Rai, R. V., Karim, A. A. 2010. Mycotoxins in Food and Feed: Present Status and Future Concerns. Comprehensive Reviews in Food Science and Food Safety, vol. 9, no. 1, p. 5781. https://doi.org/10.1111/j.1541-4337.2009.00094.X

Bhatnagar, D. K., Ehrlich, C., Cleveland, T. E. 2003. Molecular genetic analysis and regulation of aflatoxin biosynthesis. Appl. Microbiol. Biotechnol., vol. 61, no. 2, p. 8393. https://doi.org/10.1007/s00253-002-1199-x

Bhatnagar, D., Cary, J. W., Ehrlich, K., Yu, J., Cleveland, T. E. 2006. Understanding the genetics of regulation of aflatoxin production and Aspergillus flavus development. Mycopathologia; vol. 162, no. 3, p. 255-266. https://doi.org/10.1007/s11046-006-0050-9

Bilodeau, G. J. 2011. Quantitative polymerase chain reaction for the detection of organisms in soil. CAB Rev: Perspect Agr., Vet. Sci., Nutr. Natl. Res., vol. 6, no. 14, p. 1-14. https://doi.org/10.1079/PAVSNNR20116014

Bok, J. W., Keller, N. P. 2004. LaeA, a regulator of secondary metabolism in Aspergillus spp. Eukaryot Cell, vol. 3, no. 2, p. 527-535. https://doi.org/10.1128/EC.3.2.527535.2004

Boutigny, A. L., Beukes, I., Small, I., Zühlke, S., Spiteller, M., Van Rensburg, B. J., Flett, B., Viljoen, A. 2012. Quantitative detection of Fusarium pathogens and their mycotoxins in South African maize. Plant Pathol., vol. 61, no. 3 , p. 522-531. https://doi.org/10.1111/j.13653059.2011.02544.x

Brožková, I., Šmahová, P., Vytřasová, J., Mot'ková, P., Pejchalová, M., Šilha, D. 2015. Influence of chosen microbes and some chemical substances on the production of aflatoxins. Potravinarstvo, vol. 9, no. 1, p. 9-17. https://doi.org/10.5219/416

Caldas, W., Silva, S., Oliveira, J. 2002. Aflatoxinase e ocratoxina a emalimentos e riscos para a saude humana. (Aflatoxins and ochratoxin $\mathrm{A}$ in food and the risks to human health). Revista de Saude Publica, vol. 36, no. 3, p. 31932. https://doi.org/10.1590/S0034-89102002000300010

Cary, J. W., Ehrlich, K. C., Wright, M., Chang, P. K., Bhatnagar, D. 2000. Generation of aflR disruption mutants of Aspergillus parasiticus. Appl. Microbiol. Biotechnol., vol. 53, no. 6, p. 680-684. https://doi.org/10.1007/s002530000319

Cendoya, E., Monge, M. P., Palacios, S. A., Chiacchiera, S. M., Torres, A. M., Farnochi, M. C., Ramirez, M. L. 2014. Fumonisin occurrence in naturally contaminated wheat grain harvested in Argentina. Food Control, vol. 37, p. 56-61. https://doi.org/10.1016/j.foodcont.2013.09.031

Chandelier, A., Planchon, V., Oger, R. 2010. Determination of cycle cut off in real-time PCR for the detection of regulated plant pathogens. Bull. OEPP., vol. 40, no. 1, p. 52-58. https://doi.org/10.1111/j.1365-2338.2009.02354.X

Chen, R. S., Tsay, J. G., Huang, Y. F., Chiou, R. Y. 2002. Polymerase chain reaction-mediated characterization of molds belonging to the Aspergillus flavus group and detection of Aspergillus parasiticus in peanut kernels by a multiplex polymerase chain reaction. J. Food. Prot., vol. 65, no. 5, p. 840-844. https://doi.org/10.4315/0362-028X-65.5.840

Covarelli, L., Beccari, G., Prodi, A., Generotti, S., Etruschi, F., Juan, C., Ferrer, E., Mañes, J. 2015. Fusarium species, chemotype characterisation and trichothecene contamination of durum and soft wheat in an area of central Italy. Journal of the Science of Food and Agriculture, vol. 95, no. 3, p. 540-551. https://doi.org/10.1002/jsfa.6772

Creepy, E. E. 2002. Update of survey, regulation and toxic effects of mycotoxins in Europe. Toxicology Letters, vol. 127, no, 1-3, p. 19-28.

de Souza, M. P., Bittencourt, M. L., Canielles, C., Marcela, F. R., Renata, P. A., Dâmaris, S., Pérola, O. Magalhães. 2005. A biotechnology perspective of fungal proteases. African Journal of Agricultural Research, vol. 7, no. 9, p. 1368-1373, https://doi.org/10.1590/S1517-838246220140359

Dean, R., Van Kan, J. A. L., Pretorius, Z. A. 2012. The Top10 fungal pathogens in molecular plant pathology. Mol. Plant. Pathol., vol. 13, no. 4, p. 410-430. https://doi.org/10.1111/j.1364-3703.2011.00783.x

Degola, F., Berni, E., Dall'Asta, C., Spotti, E., Marchelli, R., Ferrero, I., Restivo, F. M. 2007. A multiplex RT-PCR approach 
to detect aflatoxingenic strains of Aspergillus flavus. J Appl Microbiol., vol. 203, no. 2, p. 409-427. https://doi.org/10.1111/j.1365-2672.2006.03256.x

Duan. C., Wang, X., Zhen-dong, Z. H. U., WU, X. 2007. Testing of Seedborne Fungi in Wheat Germplasm Conserved in the National Crop Genebank of China. Agric. Sci. China, vol. 6, no. 6, p. 682-687. https://doi.org/10.1016/S16712927(07)60100-X

Ehrlich, K. C., Montalbano, B. G., Cotty, P. J. 2003. Sequence comparison of aflR from different Aspergillus species provides evidence for variability in regulation of aflatoxin production. Fungal Genet. Biol., vol. 38, no. 1, p. 6374.

El-Manzalawy, Y., Dobbs, D., Honavar, V. 2008a. Predicting linear B-cell epitopes using string kernels. J. Mol. Recognit., vol. 21, no. 4, p. 243-255. https://doi.org/10.1002/jmr.893

El-Manzalawy, Y., Dobbs, D., Honavar, V. 2008b. Predicting flexible length linear B-cell epitopes. Comput. Syst. Bioinformatics Conf., vol. 7, p. 121-32. https://doi.org/10.1142/9781848162648 0011

El-Sayed, A. M. A. A. 1996. Natural occurrence of ochratoxin A and citrinin in food stuffs in Egypt. Mycotoxin Res., vol. 12, no. 1, p. 41-4. https://doi.org/10.1007/BF03192079

Fatah, S. I. A. E., Naguib, M. M., El-Hossiny, E. N., Sultan, Y., Abodalam, Y., Yli-Mattila, T. 2015. Molecular versus Morphological Identification of Fusarium spp. isolated from Egyptian corn. Research Journal of Pharmaceutical, Biological and Chemical Sciences, vol. 6, no. 4, p. 1813-1822.

Fox, E. M., Howlett, B. J. 2008. Secondary metabolism: regulation and role in fungal biology. Curr. Opin. Microbiol., vol. 11, no. $6, \quad$ p. 481-487. https://doi.org/10.1016/j.mib.2008.10.007

Frisvad, J. C., Hubka, V., Ezekiel, C. N., Hong, S. B., Nováková, A., Chen, A. J., Arzanlou, M., Larsen, T. O., Sklenár̆, F., Mahakarnchanakul, W., Samson, R. A., Houbraken, J. 2019. Taxonomy of Aspergillus section Flavi and their production of aflatoxins, ochratoxins and other mycotoxins. Stud Mycol., vol. 93, p. 1-63. https://doi.org/10.1016/j.simyco.2018.06.001

Fung, F., Clark, R. 2004. Health effects of mycotoxins: a toxicological overview. J. Toxicol. Clin. Toxicol., vol. 42, no. 2, p. 217-34. https://doi.org/10.1081/CLT-120030947

Geiser, D. M., Klich, M. A., Frisvad, J. C., Peterson, S. W., Varga, J., Samson, R. A. 2007. The current status of species recognition and identification in Aspergillus. Stud. Mycol., vol. 59, p. 1-10. https://doi.org/10.3114/sim.2007.59.01

Hussein, H. S., Brasel, J. M. 2001. Toxicity, metabolism, and impact of mycotoxins on humans and animals. Toxicology, vol. 167, no. 2, p. 101-134.

Hussien, T., Carlobos Lopez, A. L., Chris, T., Cumagum, J. C., Yli-Mattila, T. 2017. Identification and quantification of fumonisin-producing Fusarium species in grain and soil samples from Egypt and the Philippines. Phytopathologia Mediterranea, vol. 56, no. 1, p. 146-153. https://doi.org/10.14601/Phytopathol_Mediterr-20294

Ibrahim, T. F., El-Abedeen, A. Z., El-Morsy, G. A., ElAzhary, T. M. 1998. Aflatoxins in Egyptian sorghum grains: detection and estimation. J. Agric. Res., vol. 76, p. 923-931.

Ito, T., Matsui, Y., Ago, T, Ota, K., Sumimoto, H. 2001. Novel modular domain PB1 recognizes PC motif to mediate functional protein-protein interactions. $E M B O \mathrm{~J}$. vol. 20, no. 15, p 3938-3946. https://doi.org/10.1093/emboj/20.15.3938

Juan, C., Zinedine, A., Molto, J. C., Idrissi, L., Manes, J. 2008. Aflatoxins levels in dried fruits and nuts from Rabat-sale area, Morocco. Food Control, vol. 19, p. 849-853. https://doi.org/10.1016/j.foodcont.2007.08.010

Kachapulula, P. W., Akello, J., Bandyopadhyay, R., Cotty, P. J. 2017. Aspergillus section Flavi community structure in Zambia influences aflatoxin contamination of maize and groundnut. Int. J. Food Microbiol., vol. 16, no. 261, p. 49-56. https://doi.org/10.1016/j.ijfoodmicro.2017.08.014

Kolaskar, A. S., Tongaonkar, P. C. 1990. A semi-empirical method for prediction of antigenic determinants on protein antigens. FEBS Lett., vol. 276, no. 1-2, p. 172-174. https://doi.org/10.1016/0014-5793(90)80535-Q

Kovacs, M. 2004. Nutritional health aspects of mycotoxins. Orvosi Hetilap, vol. 145, no. 34, p. 1739-1746.

Kumar, V., Basu, M. S., Rajendran, T. P. 2008. Mycotoxin research and mycoflora in some commercially important agricultural commodities. Crop protection, vol. 27, no. 6, p. 891-905. https://doi.org/10.1016/i.cropro.2007.12.011

Leach, J. E., White, F. F., Rhoads, M. L., Leung, H. 1990. A Repetitive DNA Sequence Differentiates Xanthomonas campestris pv. oryzae from Other Pathovars of $X$. campestris. Mol. Plant-Microbe Interact., vol. 3, no. 4, p. 238. https://doi.org/10.1094/MPMI-3-238

Lee, T., Lee, S. H., Shin, J. Y., Yun, J. C., Lee, Y. W., Ryu, J. G. 2011. Occurrence of Fusarium mycotoxins in rice and its milling by-products in Korea. J Food Prot., vol. 74, no. 7, p. 1169-1174. https://doi.org/10.4315/0362-028X.JFP-10-564

Leslie, J. F., Summerell, B. A., Bullock, S. 2006. The Fusarium Laboratory Manual. Ames, Lowa, USA : Black well professional publishing, 388 p. ISBN: 978-0-813-81919-8. https://doi.org/10.1002/9780470278376

Lorè, A., Spadaro, D., Garibaldi, A., Gullino, M. L. 2011. Assessment of the contamination of rice grains in Piedmont by trichothecenes. Protezione delle Colture, vol. 2, p. 105-106.

Mahmoud, M. A., Al-Othman, M. R., Abd El-Aziz, A. R. M. A. 2013. Mycotoxigenic fungi contaminating corn and sorghum grains in Saudi Arabia. Pakistan J. Bot., vol. 45, no. 5, p. 1831-1839.

Makun, H. A., Gbodi, T. A., Akanya, O. H., Salako, E. A., Ogbadu, G. H. 2007. Fungi and some mycotoxins contaminating rice (Oryza sativa) in Niger State, Nigeria. Afr. J. Biotech., vol. 6, no. 2, p. 99-108.

Mwanza, M., Ndou, R. V., Dzoma, B., Nyirenda, M., Bakunzi, F. 2013. Canine aflatoxicosis outbreak in South Africa (2011): a possible multi-mycotoxins aetiology. J. S. Afr. Vet. Assoc., vol. 84, no. 1, p. E1-5.

National Library of Medicine. 2014. Basic Local Alignment Search Tool [online] s.a. [cit. 2019-01-18] Available at: http://blast.ncbi.nlm.nih.gov/

Neergaard, P. 1977. Seed pathology, vol. 1-2. Palgrave, London : MacMillan Press. ISBN: 978-1-349-02844-3. https://doi.org/10.1007/978-1-349-02842-9

Niessen, L. 2007. PCR-based diagnosis and quantification of. mycotoxin producing fungi. Int. J. Food Microbiol., vol. 119, no. $1-2, \quad$ p. $38-46$. https://doi.org/10.1016/j.ijfoodmicro.2007.07.023

Osman, N. A., Abdelgadir, A. M., Moss, M. O., Bener, A. 1999. Aflatoxin contamination of rice in the United Arab Emirates. Mycotoxin Res., vol. 15, no. 1, p. 39-44. https://doi.org/10.1007/BF02945213

Park, J. W., Choi, S. Y., Hwang, H. J., Kim, Y. B. 2005. Fungal mycoflora and mycotoxins in Korean polished rice destined for humans. Int. J. Food Microbiol., vol. 103, no. 3, p. 305-14. https://doi.org/10.1016/j.ijfoodmicro.2005.02.001

Pasquali, M., Beyer, M., Logrieco, A., Audenaert, K., Balmas, V., Basler, R., Boutigny, A. L., Chrpová, J., Czembor, E., Gagkaeva, T., González-Jaén, M. T., Vogelgsang, S. A. 
2016. European database of Fusarium graminearum and $F$. culmorum trichothecene genotypes. Frontiers in MicroBiology; vol. $7, \quad$ p. 406. https://doi.org/10.3389/fmicb.2016.00406

Passone, M. A., Rosso, L. C., Etcheverry, M. 2010. Detection and quantification of Aspergillus section flavi spp. in stored peanuts by real-time PCR of nor-1 gene, and effects of storage conditions on aflatoxin production. International Journal of Food Microbiology, vol. 138, no. 3, p. 276-281. https://doi.org/10.1016/j.ijfoodmicro.2010.01.003

Peraica, M., Rašić, D. 2012. The impact of mycotoxicoses on human history. Arh. Hig. Rada. Toksikol., vol. 63, no. 4, p. 513518. https://doi.org/10.2478/10004-1254-63-2012-2259

Pitt, J. I. 2000a. Toxigenic fungi and mycotoxins. Br. Med. Bull., vol. 56, no. 1, p. 184-92. https://doi.org/10.1258/0007142001902888

Pitt, J. I. 2000b. Toxigenic fungi: which are important? Med. Mycol., $\quad$ vol. $\quad 38, \quad$ p. $17-22$. https://doi.org/10.1080/mmy.38.s1.17.22

Probst, C., Bandyopadhyay, R., Cotty, P. J. 2014. Diversity of aflatoxin-producing fungi and their impact on food safety in sub-Saharan Africa. Int J Food Microbiol., vol. 17, no. 174, p. 113-22. https://doi.org/10.1016/j.ijfoodmicro.2013.12.010

Qiu, J. Shi, J. 2014. Genetic relationships, Carbendazim sensitivity and mycotoxin production of the Fusarium graminearumpopulations from maize, wheat and rice in eastern China. Toxins, vol. 6, no. 8, p. 2291-2309. https://doi.org/10.3390/toxins6082291

Reddy, K. R. N., Salleh, B., Saad, B., Abbas, H. K., Abel, C. A., Shier, W. T. 2010. An overview of mycotoxin contamination in foods and its implications for human health. Toxin Rev., vol. 29, no. 1, p. 3-26. https://doi.org/10.3109/15569541003598553

Richard, E., Heutte, N., Sage, L., Pottier, D., Bouchart, V., Lebailly, P., Garon, D. 2007. Toxigenic fungi and mycotoxins in mature corn silage. Food Chem. Toxicol., vol. 45, no. 12, p. 2420-2425. https://doi.org/10.1016/j.fct.2007.06.018

Sambrook, J., Fritsch, E., Maniatis, T. 1989. Molecular Cloning: A Laboratory Manual. $2^{\text {nd }}$ ed. New York, USA : Cold Spring Harbor Laboratory Press. 2028 p. ISBN-10: 0879693096.

Samina, A. 2015. Natural occurrence of Mycotoxins in Food and Feed:Pqkistan Perspective. Comprehensive Reviews in food science and food safty, vol. 1-4, no. 2, p. 159-175.

Sánchez-Hervás, M., Gil, J. V., Bisbal, F., Ramón, D., Martínez-Culebras, P. V. 2008. Mycobiota and mycotoxin producing fungi from cocoa beans. Int. J. Food Microbiol., vol. 125, no. 3, p. 336-340. https://doi.org/10.1016/j.ijfoodmicro.2008.04.021

Scauflaire, J., Mahieu, O., Louvieaux, J., Foucart, G., Renard, F., Munaut, F. 2011. Biodiversity of Fusarium species in ears and stalks of maize plants in Belgium. European Journal of Plant Pathology, vol. 131, p. 59-66. https://doi.org/10.1007/s10658-011-9787-1

Schmidt-Heydt, M. Geisen, R. 2007. A microarray for monitoring the production of mycotoxins in food. Int J Food Microbiol., vol. 117, no. 2, p. 131-140. https://doi.org/10.1016/j.ijfoodmicro.2007.01.014

Sette, A., Fikes, J. 2003. Epitope-based vaccines: An update on epitope identification, vaccine design and delivery. Curr. Opin. Immunol., vol. 15, no. 4, p. 461-470.

Shephard, G. S. 2008.Impact of mycotoxins on human health in developing countries. Food Addit. Contam. Part A Chem.
Anal. Control Expo. Risk Assess., vol. 25, no. 2, p. 146-51. https://doi.org/10.1080/02652030701567442

Singh, P., Cotty, P. J. 2019. Characterization of Aspergilli from dried red chilies (Capsicum spp.): Insights into the etiology of aflatoxin contamination. Int J Food Microbiol., vol. 16, no. 289, p. 145-153. https://doi.org/10.1016/j.ijfoodmicro.2018.08.025

Spröte, P., Brakhage, A. A., Hynes, M. J. 2009. Contribution of peroxisomes to penicillin biosynthesis in Aspergillus nidulans. Eukaryot Cell., vol. 8, no.3, p. 421-430. https://doi.org/10.1128/EC.00374-08

Suga, H., Galel, H. C. 2007. Development of VNTR markers for two Fusarium graminearum clade species. Microbiology, vol. 153 no. 6 , p. $1677-1692$. https://doi.org/10.1111/j.1471$\underline{8286.2004 .00703 . x}$

Taha, K. K., Elmahi, E. R., Hassan, H., Ahmed, S. E., Shyou, M. H. 2012. Analytical Study On Three Types Of Gum From Suda. Journal of Forest Products \& Industries, vol. 1, no. 1, p. 11-16.

Taligoola, H. K., Ismail, M. A., Chebon, S. K. 2011. Mycobiota and aflatoxins associated with imported rice grains stored in Uganda. Czech Mycol., vol. 63, no. 1, p. 93-107.

Voss, K. A., Riley, R. T., Gelineau-van, Waes. J. 2014. Fumonisin B1 induced neural tube defects were not increased in LM/Bc mice fed folate-deficient diet. Mol. Nutr. Food Res., vol. 58, no. 6, p. 1190-1198. https://doi.org/10.1002/mnfr.201300720

Yassin, M. A., El-Samawaty, A. R., Bahkali, A., Moslem, M., Abd-Elsalam, K. A., Hyde, K. D. 2010. Mycotoxinproducing fungi occurring in sorghum grains from Saudi Arabia. Fungal Divers, vol. 44, no. 1, p. 45-52. https://doi.org/10.1007/s13225-010-0058-9

Zhang, J. W., Mine, Y. 2002. Identification and fine mapping of IgG and IgE epitopes in ovomucoid. Biochem. Biophys. Res. Commun., vol. 292, no. 4, p. 1070-1074. https://doi.org/10.1006/bbrc.2002.6725

\section{Acknowledgments:}

Deanship of Scientific Research; Princess Norah bent Abdurrahman University (PNU)- project No.KG/26362. The authors are grateful to Deanship of Scientific Research; PNU for financial support. And thanks for all technicians in Research Labe, Biology department, PNU.

\section{Contact address:}

Latifa Al Husnan, Assistant Prof. Molecular Genetic, Microbiology, Princess Noruah bint Abdulrahman University (PNU), Faculty of Science, Biology department, Riyadh, Kingdom of Saudi Arabia (KSA), Tel.: +966504207002, E-mail: bio-tech-321@ @otmail.com

Muneera Al Kahtani, Associated Prof. Microbiology, Princess Noruah bint Abdulrahman University (PNU), Faculty of Science, Biology department, Riyadh, Kingdom of Saudi Arabia (KSA), Tel.: + 966- 504110897, E-mail: mdf.alkahtani@gmail.com

*Randa Mohamed Farag, Assistant prof. Microbiology, Princess Nourah bint Abdulrahman University (PNU), Health Sciences Research Center (HSCR), Riyadh, Kingdom Saudi Arabia (KSA), Tel.: +966-54-0672520, Email: randa792006@gmail.com

Corresponding author: * 